\title{
Ellagic Acid Containing Nanostructured Lipid Carriers for Topical Application: A Preliminary Study
}

\author{
Supandeep Singh Hallan $\left.{ }^{1} \mathbb{(}\right)$, Maddalena Sguizzato ${ }^{1}$, Gabriella Pavoni ${ }^{1}$, Anna Baldisserotto ${ }^{2} \mathbb{D}$, \\ Markus Drechsler ${ }^{3}\left(\mathbb{D}\right.$, Paolo Mariani ${ }^{4}\left(\mathbb{D}\right.$, Elisabetta Esposito ${ }^{1, *(\mathbb{C})}$ and Rita Cortesi ${ }^{1, *(1)}$ \\ 1 Department of Chemical and Pharmaceutical Sciences, University of Ferrara, 44121 Ferrara, Italy; \\ hllsnd@unife.it (S.S.H.); sgzmdl@unife.it (M.S.); gabriellapavoni@tiscali.it (G.P.) \\ 2 Department of Life Sciences \& Biotechnology, University of Ferrara, 44121 Ferrara, Italy; bldnna@unife.it \\ 3 Bavarian Polymerinstitute (BPI), University of Bayreuth, 95440 Bayreuth, Germany; \\ Markus.Drechsler@uni-bayreuth.de \\ 4 Department of Life and Environmental Sciences, Polytechnic University of Marche, 60131 Ancona, Italy; \\ p.mariani@staff.univpm.it \\ * Correspondence: ese@unife.it (E.E.); crt@unife.it (R.C.); Tel.: +39-0532-455-259 (R.C.)
}

Academic Editors: Fabio Sonvico, Yangchao Luo and Domenico Lombardo Received: 12 February 2020; Accepted: 18 March 2020; Published: 23 March 2020

Abstract: Ellagic acid (EA) is a potent antioxidant substance of natural origin characterized by poor biopharmaceutical properties and low solubility in water that limit its use. The aim of the present study was to develop lipid-based nanoparticle formulations able to encapsulate EA for dermal delivery. The EA-loaded nanoparticles were prepared using two different lipid compositions, namely tristearin/tricaprylin (NLC-EA1) and tristearin/labrasol (NLC-EA2). The influence of formulations on size, entrapment efficiency, and stability of EA-loaded nanoparticles was investigated. Cryo-TEM and small-angle X-ray scattering (SAXS) analyses showed that no morphological differences are evident among all the types of loaded and unloaded nanostructured lipid carriers (NLCs). The macroscopic aspect of both NLC-EA1 and NLC-EA2 did not change with time. No difference in size was appreciable between empty and drug-containing NLC, thus the nanoparticle diameter was not affected by the presence of EA and in general no variations of the diameters occurred during this time. The entrapment efficiency of both EA-loaded nanoparticles was almost quantitative. In addition, NLC-EA1 maintained EA stability for almost two months, while NLC-EA2 up to 40 days. FRAP (Ferric reducing ability of plasma) assay showed an antioxidant activity around $60 \%$ for both the loaded NLC, as compared to the solution. Although both types of NLC are characterized by some toxicity on $\mathrm{HaCaT}$ cells, NLC-EA1 are less cytotoxic than NLC-EA2. Taken together these results demonstrated that the inclusion of EA within NLC could improve the water solubility, allowing for a reduction of the dosage. Moreover, both types of NLC-EA maintained a high antioxidant effect and low toxicity.

Keywords: nanostructured lipid carriers (NLCs); lipid-based nanosystems; phytopharmaceutics; ellagic acid; antioxidant activity

\section{Introduction}

Phytopharmaceuticals are pharmaceuticals derived from botanicals. Ellagic acid (EA) is a phytopharmaceutical substance found in many fruits and plants such as raspberries, strawberries, pomegranates, blackberries, and many other plant foods. EA (2,3,7,8-tetrahydroxy-chromeno [5,4,3-cde]chromene-5,10-dione) (Table 1) is the dimeric derivative of gallic acid and has a significant attractiveness in food supplements because of its potentially beneficial effects against a wide range of diseases [1]. Various studies indicate that EA possesses antimutagenic, antiagenic, antioxidant, and anti-inflammatory activity in bacterial and mammalian systems [2-4]. In addition, 
EA has proven to be an efficient skin whitener and suppressor of pigmentation. In addition to its anti-oxidant activities, EA also is cytotoxic towards different types of cancer cells, such as osteogenic sarcoma, tongue, pancreatic, leukemic, neuroblastoma, breast, prostate gland, and colon cells [5-11], and possesses anti-inflammatory, anti-bacterial, anti-angiogenesis, anti-atherosclerosis, anti-hyperglycemic, antihypertensive, and cardioprotective effects [1,12-14]

Table 1. Chemical structure and some physicochemical characteristics of ellagic acid (EA).

\begin{tabular}{lllll}
\hline Chemical Structure & Molecular Weight & $\lambda_{\max }(\mathrm{nm})$ & $\log \mathrm{P}$ & Melting Point $\left({ }^{\circ} \mathrm{C}\right)$ \\
\hline
\end{tabular}

However, EA has found limited use in therapeutic applications due to its low water solubility (around $9.7 \mu \mathrm{g} / \mathrm{mL}$ ) and permeability (class IV of the Biopharmaceutics Classification System) [15,16]. When orally administered EA is poorly absorbed due to low aqueous solubility, metabolism in the gastrointestinal tract [17], first pass effect and irreversible binding to cellular DNA and proteins problems.

Incorporation of drugs in lipid nanoparticles is a smart approach to overcome bioavailability [18]. Among lipid-based colloids, solid lipid nanoparticles (SLNs) and nanostructured lipid carriers (NLCs) can be mentioned. SLNs are generally produced using solid lipids stabilized by the presence of surfactants dispersed in the aqueous phase. As a drawback, during lipid crystallization of the solid lipid core of these particles may lead to leakage of the included active compound, thus influencing the encapsulation efficiency. NLCs were developed to overcome these drawbacks by mean of a partial replacement of the solid lipid with a fluid lipid. In this way the lipid core matrix becomes less ordered as compared to SLNs and can accommodate high amounts of active compound reducing loss problems.

The main advantages of both SLNs and NLCs are their ability to incorporate active compounds, improve stability and bioavailability of the entrapped molecules, possibly control release and targeting together with safety, low cost of production, and easy scaling-up. Due to their great versatility, biodegradability, biocompatibility, and targeting, the lipid-based nanoparticles have been used for several administration routes, such as oral, parenteral, ocular [19], and topical [20,21]. In particular, topical administration of active compounds loaded onto SLNs or NLCs could prevent their systemic absorption and hence, side effects. Furthermore, their small size ensures a close contact with the stratum corneum, facilitating drug skin penetration.

On the other hand, common disadvantages of SLN include particle aggregation, particle growth, unexpected dynamics of polymorphic transition, burst drug release, and low incorporation capacities. Concerning this last drawback, it can be underlined that triglycerides are known to crystallize mainly in three polymorphic forms which transform monotrophically from $\alpha$, via $\beta^{\prime}$, to $\beta$ [22]. During storage, SLN triglycerides are subjected to a shift into low energy and more ordered $\beta$ modification, which cause a reduction of imperfections in the crystal lattice and consequently, drug expulsion. When SLNs are formulated with mono acid glyceride, such as tristearin, the drug loading is limited, and drug expulsion occurs within short times.

Instead, NLCs are composed of a solid lipid matrix with a certain content of a liquid lipid phase able to better solubilize drugs as compared to solid lipids. The liquid lipids form droplets within the solid lipid particles matrix, providing a high incorporation capacity and a control of drug release. Therefore, NLCs, which are solid but not crystalline, overcome the drawback of drug expulsion. Indeed, the use of mixtures of solid and liquid lipids allows for the obtaining of nanoparticles that become solid 
after cooling but do not crystallize [23]. In these structures, the oily nano-compartments are surrounded by a solid matrix and the solid matrix of the lipid nanoparticle contains tiny liquid nano-compartments of oil; this peculiar lipid organization increases drug solubility, leading to an enhancement of the drug loading capacity. In addition, analyzing the production methods of SLNs, it was observed that too high of a concentration of the drug in the molten lipidic phase could lead to an immediate drug expulsion during the cooling process or to a dilution in the cold water.

For our studies, we analyzed two different blends of solid and liquid lipids. The first one was based on the use of a mixture of the liquid caprylic/capric triglycerides (Miglyol) and the solid tristearin. In the second case, a mixture of caprylocaproyl macrogol-8 glyceride (Labrasol) and tristearin was used to produce NLCs. In the hot state the two lipids form one phase and during the cooling process a phase separation occurs, leading to inclusion of small oily droplets in the solid matrix. Taking into account these assumptions, the present paper will describe the preparation, characterization, and preliminary in vitro studies of EA-containing NLCs for dermatologic purposes.

\section{Results and Discussion}

\subsection{Production and Characterization of NLC-EA Dispersions}

The NLC-EA composed as reported in Table 2, were obtained by dispersing the lipid phase in the aqueous phase under sonication [23] achieving stable and homogenous dispersions. The corresponding empty NLC, namely e-NLC1 and e-NLC2, were obtained using the same procedure apart for the addition of EA. As already known, a fluid lipid (i.e., tricaprylin or labrasol) when mixed to solid lipids allows for the formation of solid particles homogenously embedded with fluid compartments. However, during the preparation almost $4 \%$ by weight of total used lipid phase was lost on the vessel and less than $1 \%$ gave rise to the formation of agglomerates.

Table 2. Composition of the produced nanostructured lipid carriers (NLC)-EA.

\begin{tabular}{ccc}
\hline & $\begin{array}{c}\text { NLC-EA1 } \\
\text { (\% of Total Dispersion's Weight) }\end{array}$ & $\begin{array}{c}\text { NLC-EA2 } \\
\text { (\% of Total Dispersion's Weight) }\end{array}$ \\
\hline Tristearin & 3.35 & 4 \\
Miglyol & 1.65 & - \\
Labrasol & - & 1 \\
Aqueous Solution of & 95 & 95 \\
Poloxamer 188 (2.5\% $w / v)$ & 0.025 & 0.025 \\
Ellagic Acid (EA) & & \\
\hline
\end{tabular}

After production, nanoparticles were characterized in terms of dimensions and morphology. Dimensions and size distribution of the produced NLCs were determined by mean of photon correlation spectroscopy (PCS). The analyses were made immediately after preparation and periodically at regular intervals in order to investigate the stability of nanoparticles by time. Table 3 summarizes the obtained values of mean diameters and polydispersity.

Analyzing the obtained values, it should be noted that in general no variations of the diameters occurred during this time. Some differences in size are appreciable between empty and drug-containing nanoparticles only in the case of NLC1, which shows an increase from $116.5 \mathrm{~nm}$ to $195.7 \mathrm{~nm}$ in the presence of EA. However, concerning the polydispersity indexes, empty NLCs showed a reduction during time while EA-loaded NLCs showed no great variations, indicating that NLCs maintain up to two months their monomodal dimensional distribution. 
Table 3. Mean diameters of NLCs as determined by photon correlation spectroscopy (PCS).

\begin{tabular}{ccccc}
\hline \multirow{2}{*}{ Day } & e-NLC1 & e-NLC2 & NLC-EA1 & NLC-EA2 \\
\cline { 2 - 5 } & Z ave $(\mathbf{n m})$ & Z ave $(\mathbf{n m})$ & Z ave $(\mathbf{n m})$ & Z ave $(\mathbf{n m})$ \\
& $\boldsymbol{P . I .}$ & $\boldsymbol{P . I .}$ & $\boldsymbol{P . I .}$ & $\boldsymbol{P . I .}$ \\
\hline \multirow{2}{*}{1} & $116.5 \pm 0.6$ & $188.3 \pm 1.2$ & $195.7 \pm 2.1$ & $189.6 \pm 3.9$ \\
& 0.37 & 0.31 & 0.36 & 0.33 \\
\hline \multirow{2}{*}{20} & $118.9 \pm 5.6$ & $183.1 \pm 6.2$ & $192.0 \pm 0.8$ & $181.3 \pm 5.1$ \\
& 0.40 & 0.31 & 0.52 & 0.29 \\
\hline \multirow{2}{*}{30} & $117.2 \pm 7.6$ & $182.0 \pm 1.5$ & $190.4 \pm 2.9$ & $195.8 \pm 6.4$ \\
& 0.43 & 0.28 & 0.40 & 0.37 \\
\hline \multirow{2}{*}{60} & $118.7 \pm 2.3$ & $176.6 \pm 3.2$ & $189.8 \pm 2.3$ & $189.2 \pm 2.6$ \\
& 0.39 & 0.33 & 0.30 & 0.31 \\
\hline
\end{tabular}

s.d. = standard deviation calculated after five determinations on different batches of the same type of dispersion. P.I.: polydispersity index

Cryo-transmission electron microscopy allows for the study of the morphology of the produced NLCs. Figure 1 depicts the cryo-TEM images of e-NLC1, e-NLC2, NLC-EA1, and NLC-EA2. It is evident that no morphological differences are evident among the considered types of NLCs. Notably, some large and flat particles are detectable together with deformed, elongated, and circular platelet-like or elliptical shaped nanoparticles depending on their position with respect to the site of observation, namely from the top to edge-on view or intermediate positions $[24,25]$.
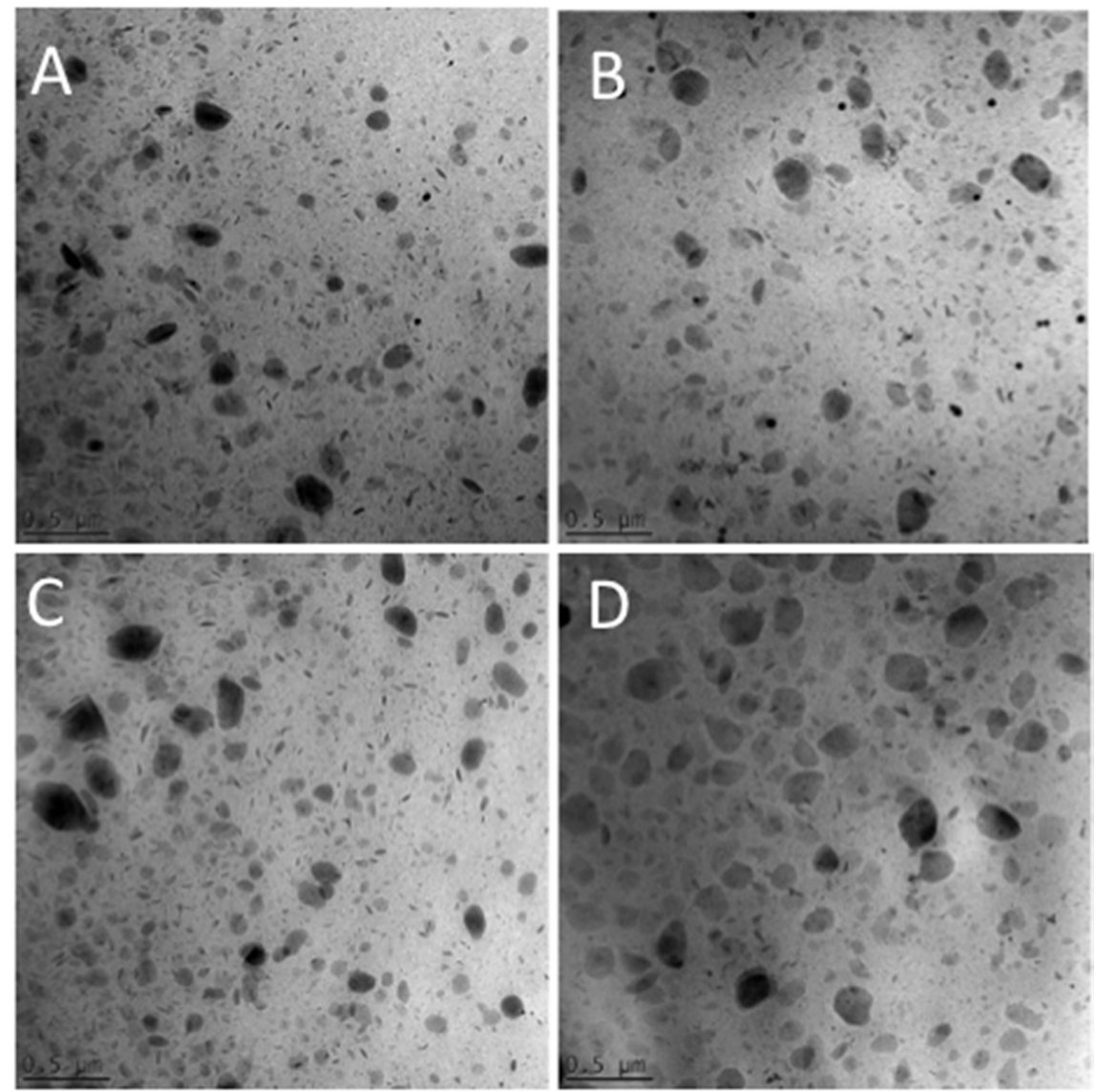

Figure 1. Cryo-transmission electron microscopy (cryo-TEM) images of e-NLC1 (A), NLC-EA1 (B), e-NLC2 (C), and NLC-EA2 (D). 
The inner structural features of NLC, NLC-EA1, and NLC-EA2 were investigated by small-angle X-ray scattering (SAXS). Results are reported in Figure 2 and clearly indicate that NLC-EA1 and NLC-EA2 nanoparticles are characterized by a lamellar organization of the inner matrix, both in the presence or absence of EA. Particularly, SAXS profiles show a Bragg peak at $Q=0.143 \AA^{-1}$, which corresponds to a lamellar structure with a repeat distance (which measures the sum of the bilayer thickness and the thickness of the water layer separating two adjacent bilayers) of $43.9 \AA$. Indeed, the four preparations are very similar, therefore it can be asserted that neither the different lipid composition nor the presence of EA modify the structural organization of lipid nanoparticles. Note that considering the very low solubility of EA in water, data suggests a solubilization of the drug inside the paraffinic region of the lipid layer.

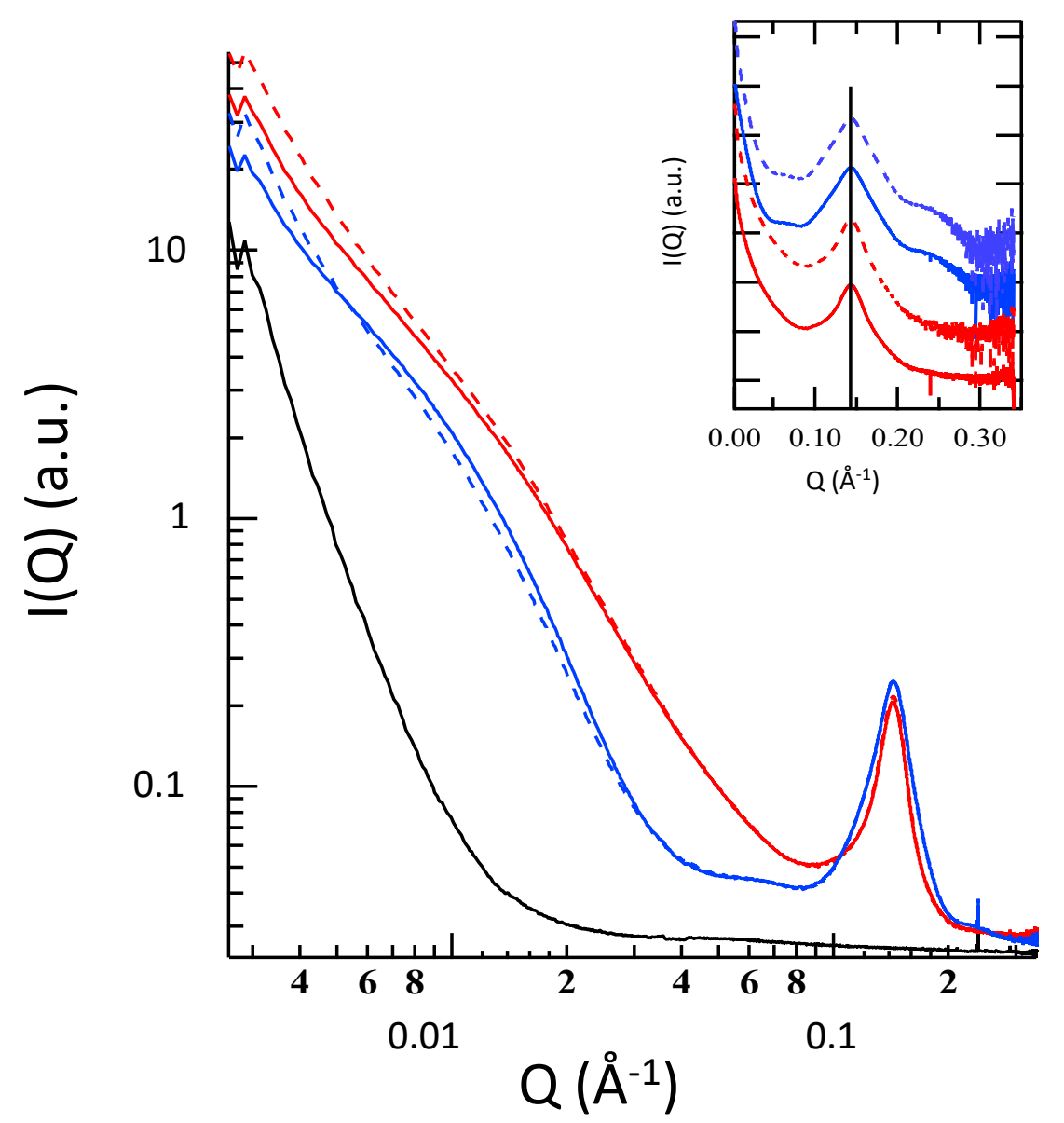

Figure 2. Small-angle X-ray scattering (SAXS) profiles of e-NLC1 (full blue line), e-NLC2 (full red line), NLC-EA1 (dotted blue line), NLC-EA2 (dotted red line). The signal of sole water is indicated with a black line. The inset shows the SAXS profiles after the subtraction of the water contribution. Here, the curves are scaled for clarity. The vertical black line indicates the constant position of the Bragg peaks.

\subsection{Efficiency of Drug Encapsulation and Shelf-Life}

The amount of drug encapsulated in NLCs with respect to the total amount used for the preparation, was evaluated by high-performance liquid chromatography (HPLC) using a reversed-phase column as described in the experimental section. The amount of entrapped EA was determined after centrifugation by dissolving in a known amount of ethanol the lipid phase, while the amount of free EA was determined in the filtrated aqueous phase. The content of EA in both fractions was calculated by comparison with 
a standard solution. Particularly, EA content in the produced NLCs was calculated as a function of time and expressed as a percentage of the total amount used for the preparation.

As reported in Table 4, drug recovery after NLC production was almost quantitative as compared to the total amount used for the preparation. In the aqueous fraction the amount of EA was always below the detection limit of the analytical method employed, indicating a high encapsulation efficiency of loaded NLC. Indeed, the drug encapsulation was $91.50 \pm 2.42 \%$ in the case of NLC-EA1 and $96.61 \pm 3.67 \%$ in the case of NLC-EA2 dispersions.

Table 4. EA content in NLCs as a function of time and shelf-life values.

\begin{tabular}{cccc}
\hline \multicolumn{4}{c}{ EA Recovery (\%) $\mathbf{1}$} \\
\hline Time (Days) & EA (Ethanol Solution) & NLC-EA1 & NLC-EA2 \\
\hline 1 & $100.00 \pm 1.41$ & $91.50 \pm 2.42$ & $96.61 \pm 3.67$ \\
10 & $96.58 \pm 2.22$ & $91.06 \pm 3.91$ & $92.69 \pm 0.93$ \\
20 & $84.31 \pm 2.81$ & $90.37 \pm 2.50$ & $91.15 \pm 2.27$ \\
30 & $74.29 \pm 2.63$ & $89.66 \pm 2.51$ & $90.86 \pm 1.85$ \\
60 & $48.24 \pm 6.14$ & $87.57 \pm 2.40$ & $88.63 \pm 3.67$ \\
\hline \multicolumn{4}{c}{ Shelf Life Values } \\
$t_{90}($ days) & ${ }^{2}$ & 0.012325 & 0.001832 \\
$t_{1 / 2}($ days) & 3 & 5.52 & 57.30 \\
\hline
\end{tabular}

${ }^{1}$ : percentage as a function of initial EA content by weight. ${ }^{2}$ : time at which the drug concentration has lost $10 \% .{ }^{3}$ : time at which the drug concentration has lost $50 \%$. The results are the average of three independent experiments \pm s.d.

Shelf life stability was calculated plotting Log (EA residual content, \% with respect to drug content at time 0 ) against time, obtaining first order kinetics (data not shown). From the slopes (m) obtained by linear regression, the time at which the drug concentration lost $10 \%$ and $50 \%$, namely shelf life $\left(t_{90}\right)$ and half-life $\left(t_{1 / 2}\right)$ respectively, was calculated and reported in Table 4 . All data were statistically significant $(p<0.0001)$.

It was found that EA in solution decomposes quickly ( $t_{1 / 2}$ being 56 days), while NLCs are able to increase the protection of EA as compared to the solution with different efficiency. Indeed NLC-EA1 maintain $90 \%$ of EA stability for almost two months (57 days), whilst for NLC-EA2, $t_{90}$ is around 40 days. The $t_{1 / 2}$ values reach more than one year for NLC-EA1 (378 days) and 8.5 months for NLC-EA2, increasing the stability of EA 6.72- and 4.59-fold as compared to the solution.

The macroscopic aspect of both NLC-EA1 and NLC-EA2 did not change by time. Notably, no phase separation phenomena, settling of particles, and aggregate formation were evident after three months from production.

\subsection{In Vitro Experiments}

\subsubsection{Antioxidant Activity}

It is well known that various pathophysiological processes are due to the presence of free radicals, thus the antioxidant intervention is of pivotal importance. Indeed, the beneficial effects of polyphenols on human skin are largely described, such as antioxidant, anti-aging, anti-inflammatory and anti-cancer activities [26-28]. In this view, both EA-containing NLC formulations were subjected to two different tests to evaluate their antioxidant capacity as compared to the active solution and the empty NLC. The chosen tests were DPPH (2,2-Diphenyl-1-picrylhydrazyl) and FRAP (Ferric reducing ability of plasma).

It has to be underlined that in both cases it was impossible to test the activity of empty NLCs because the addition of their dimethyl sulfoxide (DMSO) solution to the radical or FRAP mixture gave rise to the formation of a certain opalescence or precipitation, respectively. As for the NLCs containing EA, the data summarized in Table 5 relate to extremely diluted solutions that did not give 
great opalescence or precipitation problems in the test media. However, unlike EA that is perfectly soluble in the chosen solvent (DMSO), the two NLCs in the same solvent appeared as suspensions.

Table 5. Antioxidant activity of NLC-EA as determined by FRAP and DPPH assays.

\begin{tabular}{|c|c|c|c|c|}
\hline \multirow{2}{*}{ Compound * } & \multicolumn{2}{|c|}{ DPPH } & \multicolumn{2}{|c|}{ FRAP } \\
\hline & $\mu \mathrm{molTE} / \mathrm{g}^{\mathrm{a}} \pm \mathrm{SD}$ & $\%$ of Activity ${ }^{b}$ & $\mu \mathrm{molTE} / \mathrm{g}^{\mathrm{a}} \pm \mathrm{SD}$ & $\%$ of Activity ${ }^{b}$ \\
\hline EA-solution & $25834.90 \pm 0.00$ & 100 & $34052.21 \pm 1902.66$ & 100 \\
\hline NLC-EA1 & $9545.16 \pm 0.00$ & 37 & $19852.21 \pm 1419.89$ & 58 \\
\hline NLC-EA2 & $4786.36 \pm 112.17$ & 18 & $20879.52 \pm 1981.78$ & 61 \\
\hline
\end{tabular}

* all the compounds were tested at the same concentration $(0.005 \mathrm{mg} / \mathrm{mL})^{\mathrm{a}}: \mu \mathrm{mol}$ Trolox equivalents/g $\mathrm{b}: \%$ of activity $=$ percentage of antioxidant activity as compared to EA in DMSO solution.

In light of these observations, the antioxidant activity data reported in Table 5 indicate for both types of NLC-EA a lower activity with respect to EA solution. In particular, regarding the FRAP assay, the antioxidant activity of both loaded NLCs is around $60 \%$ as compared to the solution. This behavior could be possibly ascribed to a combination of events. Firstly the recovered amount of EA within the formulation is lower as compared to the solution being comprised between $91 \%-96 \%$ (Table 4 ) and the amount of solubilized EA in the reaction environment. More precisely, the EA dispersed within solid NLCs can be in solid form, therefore it is not readily available in the useful form to express the antioxidant activity. In other words, there is a lag time in which EA must pass from the solid to the solubilized form in solution capable of interacting with ferric ions. Thus, these conditions may affect the actual EA concentrations useful for the determination of antioxidant activity, but in the meantime they give good results about the potential activities of these formulations. Indeed, the rate of EA dissolution could possibly influence a lasting antioxidant activity during time. On the other hand, the values obtained with the DPPH assay are quite far from those expected, certainly due to the incompatibility of the DMSO solvent with the test methodology [29,30]. The profile emerged from these tests underlines how the two new formulations preserve the excellent antioxidant capacity of the active.

\subsubsection{EA Diffusion from NLC}

To evaluate the release of EA from NLC-EA1 and NLC-EA2 formulations, Franz-cells associated to nylon membrane were used. Particularly, two different $\mathrm{pH}$ values were considered for the receiving phase constituted of phosphate buffer, namely pH 7.4 and 5.5. Furthermore, to establish the sink conditions and promote EA solubilization, 30\% ethanol by volume was added to the receiving phase [31,32]. Moreover, it has to be underlined that due to the poor water-solubility of EA, the comparative EA solution used for diffusion release experiments was made in DMSO.

Figure 3 reports the diffusion release profiles of EA from solution and both types of NLCs. The amount of EA that penetrated through the membrane per unit area was plotted against time and the slopes, which represent the steady state fluxes, and were calculated by linear regression. The calculated regression coefficients squared were higher than 0.96 . The slopes were then substituted into Equation (6) for the determination of normalized fluxes (Jn) and the results of these calculations are reported in Table 6.

Particularly, in agreement with the scarce water solubility of EA, the Jn values were in general very low at 0.003 and 0.663 . Notwithstanding these results, from the obtained profiles it is evident that the two NLC systems displayed a similar behavior in controlling EA release. Moreover, as expected, the influence of the $\mathrm{pH}$ on the receiving phase is appreciable. Indeed, as indicated by literature, $\mathrm{pH}$ influences the release, solubility, and permeation of acidic drugs [33-36]. It is interesting to see that at $\mathrm{pH}$ typical of skin surface, which ideally should be slightly acidic being comprised in the acidic range from $\mathrm{pH} 4.0$ to 7.0 [33], the release of EA is higher as compared to the same formulation tested at 
neutral $\mathrm{pH}$ (i.e., $\mathrm{pH}$ 7.4) (see Figure 3 and Table 5). This result could be noteworthy for a potential topical application of NLC-EA onto the skin.

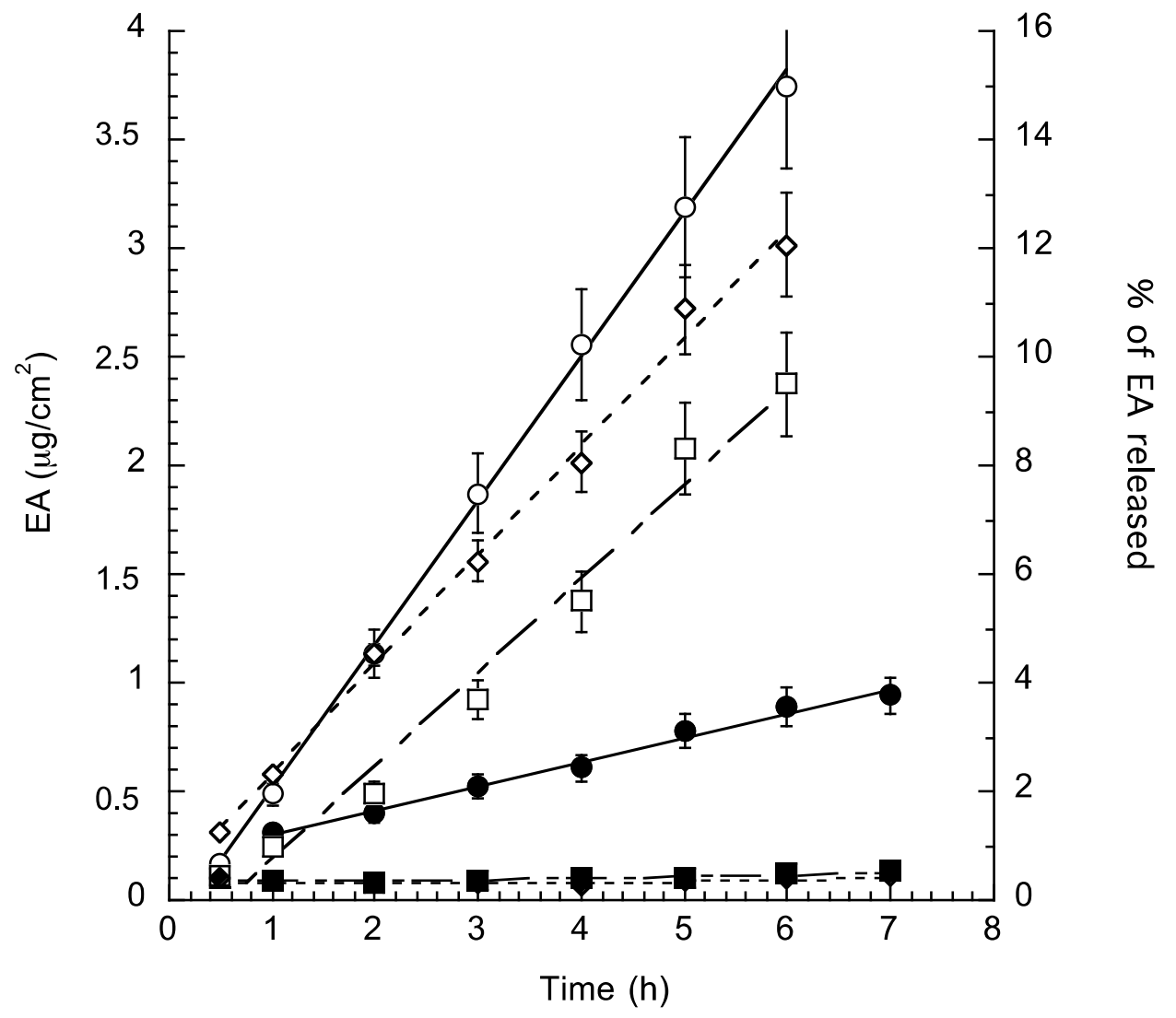

Figure 3. In vitro diffusion kinetics of EA from DMSO solution (circles) or NLC-EA1 (squares) and NLC-EA2 (diamonds) as determined by Franz cells associated to nylon membranes. Experiments were conducted in phosphate buffer at different pH, namely 7.4 (closed symbols) and 5.5 (open symbols). Data are the mean of four independent experiments \pm s.d.

Table 6. In vitro diffusion coefficients of EA.

\begin{tabular}{cccc}
\hline & Jn $\left(\mu \mathbf{g} / \mathbf{c m}^{\mathbf{2}} \mathbf{h}\right)$ & $\log \mathbf{~} \mathbf{n}$ & $\mathbf{R}^{\mathbf{2}}$ \\
\hline NLC-EA1 pH 7.4 & 0.005 & -2.30 & 0.969 \\
NLC-EA2 pH 7.4 & 0.003 & -2.52 & 0.984 \\
EA-solution pH 7.4 & 0.112 & -0.95 & 0.990 \\
NLC-EA1 pH 5.5 & 0.430 & -0.36 & 0.979 \\
NLC-EA2 pH 5.5 & 0.500 & -0.30 & 0.995 \\
EA-solution pH 5.5 & 0.663 & -0.17 & 0.999 \\
\hline
\end{tabular}

\subsubsection{Cytotoxicity Studies}

It is well known that lipid nanocarriers can improve solubilization and stabilization of drug molecules, thus influencing the pharmacokinetics of drugs in reason of the different distribution after systemic administration [37]. Moreover, lipids are physiological safe compounds as components of many natural food sources and therefore present metabolic pathways for their degradation. In addition, due to the promising results concerning EA diffusion and antioxidant activity, the in vitro activity of the produced formulation was further investigated. Particularly, cytotoxicity was assessed by the colorimetric MTT assay (3-(4,5-dimethylthiazol-2-yl)-2,5- diphenyltetrazolium bromide) on HaCaT cells comparing the activity of EA-loaded NLCs to that of EA in DMSO solution. The obtained results are graphically shown in Figure 4. 


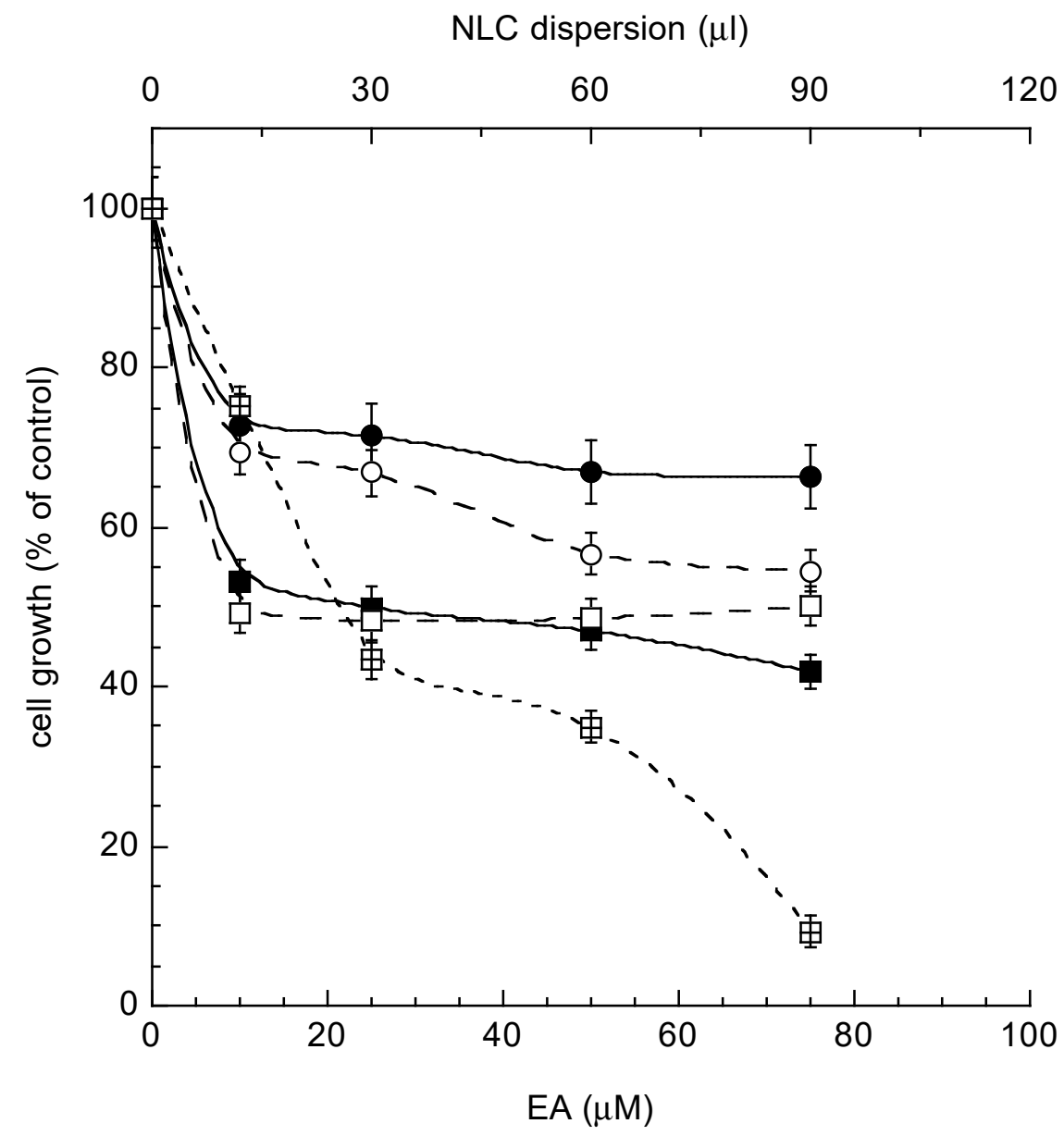

Figure 4. In vitro antiproliferative effect on HaCaT cells of EA in DMSO solution (crossed squares), e-NLC1 (closed circles), e-NLC2 (closed squares), NLC-EA1 (open circles), and NLC-EA2 (open squares). Data are the mean of three independent experiments \pm s.d. conducted in triplicate. $p$-values are always $<0.01$.

It should be underlined that, although both types of NLCs are characterized by some toxicity, NLC1 are less cytotoxic than NLC2. Furthermore, the presence of EA does not heavily influence the cytotoxicity of these formulations. On the other hand, a result that confirms our initial hypothesis is that both these formulations have a reduced cytotoxicity as compared to that of the DMSO solution, allowing us to propose them as a possible vehicle for EA.

\section{Materials and Methods}

\subsection{Materials}

Ellagic acid was purchased from Sigma-Aldrich. Miglyol 812N (tricaprylin; C8/C10 fatty acid triglycerides; caprylic/capric triglycerides; 1,2,3-propanetriyl ester caprylic acid; caprylic acid, 1,2,3-propanetriyl ester; glycerol trioctanoate; glyceryl tricaprylate; octanoic acid, 1,2,3propanetriyl ester) was a gift of Cremer Oleo Division (Witten, Germany). Labrasol ${ }^{\circledR}$ (caprylocaproyl macrogol-8 glyceride; PEG-8 caprylic/capric glycerides) was purchased form Gattefossé (Saint-Priest, France). Tristearin (Propane-1,2,3-triyltrioctadecanoate; 1,3-Di(octadecanoyloxy)propan-2-yloctadecanoate; stearic triglyceride; glyceryl stearate), poloxamer 188 (methyl-oxirane polymer, 75:30), and all other solvents and materials were provided by Merck (Milano, Italy). 


\subsection{NLC Preparation}

NLCs were prepared by hot homogenization and ultrasonication. Shortly, a lipid mixture composed as reported in Table 2 constituting the $5 \%$ by weight as compared to the total weight of dispersions, was melted at $70{ }^{\circ} \mathrm{C}$. Then poloxamer 188 aqueous solution $(2.5 \% w / w)$ was added at the same temperature under 15,000 rpm homogenization for $1 \mathrm{~min}$ (Ultra Turrax T25, IKA-Werke GmbH \& Co. KG, Staufen, Germany). Afterwards the obtained emulsion was ultrasonicated (MicrosonTM, Ultrasonic cell Disruptor) at $7 \mathrm{kHz}$ for $15 \mathrm{~min}$ and cooled down to room temperature.

EA-containing NLCs (NLC-EA) were prepared by adding a DMSO solution of the drug to the molten lipid mixture at $70^{\circ} \mathrm{C}$. Afterwards the production protocol proceeded with the addition of the poloxamer aqueous phase as above described. NLC dispersions were stored at room temperature.

\subsection{NLC Characterization}

\subsubsection{Cryo-Transmission Electron Microscopy (Cryo-TEM)}

Samples of $2 \mu \mathrm{l}$ droplets were vitrified on a lacey carbon filmed copper grid (Science Services, Munich), by insufflation of the mean of air plasma glow discharge (Solarus 950, Gatan Inc., Munich, Germany) for $30 \mathrm{~s}$. After removing the liquid by means of blotting paper, the specimen was frozen by immersion into refrigerated liquid ethane at approximately $90 \mathrm{~K}$ in a temperature-controlled freezing unit (LEICA EM GP, Wetzlar, Germany). The vitrified specimen was transferred to a Zeiss/LEO EM922 transmission electron microscope for imaging using a cryoholder (CT3500, Gatan Inc., Munich, Germany). The temperature of the sample was kept around $90^{\circ} \mathrm{K}$ throughout the examination. Specimens were examined under doses of about 100-1000 e/nm $\mathrm{nm}^{2}$ at $200 \mathrm{kV}$. Images were recorded digitally by a bottom mounted CCD camera (Ultrascan 1000, Gatan, Pleasanton, CA, USA) and subjected to image processing using the system Digital Micrograph GMS 1.9 (Gatan, Munich, Germany).

\subsubsection{Photon Correlation Spectroscopy (PCS)}

Submicron particle size analysis was performed using a Zetasizer Nano S90 (Malvern Instr., Malvern, England) equipped with a $5 \mathrm{~mW}$ helium neon laser with a wavelength output of $633 \mathrm{~nm}$. Plastic-ware was cleaned with detergent washing and rinsed twice with milliQ water. Measurements were made at $25^{\circ} \mathrm{C}$ at an angle of $90^{\circ}$. Data were interpreted using the "method of cumulants" [38].

\subsubsection{Small Angle X-rays Scattering (SAXS)}

Small-angle X-ray scattering (SAXS) data were collected on the bioSAXS beamline B21, at Diamond Light Source (Harwell, UK).

EA loaded and unloaded NLC1 and NLC2 $(6 \mathrm{mg} / \mathrm{mL})$ solutions were transferred into $0.2 \mathrm{~mL}$ tubes in an automated sample changer. The samples were then delivered into a temperature-controlled quartz capillary and exposed for $1 \mathrm{~s}$, acquiring 30 frames at $20^{\circ} \mathrm{C}$. Data were collected using a Pilatus Dectris $2 \mathrm{M}$ detector with a $3.9 \mathrm{~m}$ sample-detector distance and X-ray wavelength $\lambda=1.0 \AA$ (so that the explored Q-range extended from 0.003 to $0.35 \AA^{-1}$, Q being the modulus of the scattering vector, defined as $4 \pi \sin \theta / \lambda$, where $2 \theta$ is the scattering angle) and corrected for background, detector efficiency, and sample transmission. The two-dimensional (2D) data were then radially averaged to derive $\mathrm{I}(\mathrm{Q})$ vs. Q curves.

\subsection{Drug Content of Dispersions}

The total drug content (free plus bonded) of the produced NLC dispersions was determined after subjecting a sample of NLCs to methanol dilution (1:10 v/v) and a $3 \mathrm{~h}$ of stirring, thus destroying the lipid nanoparticles completely. On the other hand, the encapsulation efficiency (EE) of NLCs was determined as follows. One hundred microliters aliquot of each batch was loaded in a centrifugal filter (Microcon centrifugal filter unit YM-10 membrane, NMWCO 10 kDa, Sigma Aldrich, St Louis, 
MO, USA) and centrifuged (Spectrafuge ${ }^{\mathrm{TM}}$ 24D Digital Microcentrifuge, Woodbridge, NJ, USA) at 8000 rpm for $20 \mathrm{~min}$. The amount of entrapped EA was determined by dissolving the lipid phase in the supernatant with a known amount of ethanol $(1: 10, v / v)$, while the amount of free EA was determined in the filtrated aqueous phase. The samples were then filtered through $0.45 \mu \mathrm{m}$ membrane pore size and analyzed by HPLC as detailed below. All data were the mean of 6 determinations on different batches of the same type of dispersion. EE was determined applying the following equation.

$$
\mathrm{EE}=\text { amount of EA detected in the lipid phase } \times 100 / \text { total amount of EA employed }
$$

HPLC determinations were performed using an HPLC system Series 1200 (Agilent Technologies Italia, Milan, Italy) equipped with a two-plungers alternative pump (Jasco Corporation, Cremella, Italy) and an UV-detector at $254 \mathrm{~nm}$. Then, $40 \mu \mathrm{L}$ samples were injected by means of a 7125 Rheodyne injection valve with a $50 \mu \mathrm{L}$ loop on a stainless steel Kinetex ${ }^{\circledR} \mathrm{C} 18$ reverse-phase column $(150 \mathrm{~mm} \times 4.6 \mathrm{~mm})$ packed with $5 \mu \mathrm{m}$ particles (Phenomenex Srl, Milan, Italy). Injections were repeated thrice. Elution of EA was performed with a mobile phase containing methanol (55\%), water $(45 \%)$, and phosphoric acid $(0.1 \%)$ flowing at a rate of $0.6 \mathrm{~mL} / \mathrm{min}$. In these conditions, EA retention time was $5.9 \mathrm{~min}$.

\subsection{Shelf-Life Studies}

Shelf-life stability studies were conducted in triplicate by analyzing at predetermined times, the physical aspect, the drug entrapment, and the size of NLC dispersions up to 2 months from production.

Particularly, physical stability studies were performed analyzing macroscopic aspect (phase separation, turbidity, and macroscopic viscosity) under visual inspection; the drug entrapment was analyzed by HPLC as above described while the size was followed by PCS analyses as above indicated.

Chemical stability was evaluated determining EA content by HPLC analyses (see above), thus shelf-life values were calculated as described by Pugh [39] and summarized below.

$\log$ (EA residual content, \%) was plotted against time allowing the calculation of the slopes $(\mathrm{m})$ by linear regression. Afterwards, the slopes $(\mathrm{m})$ values were used for the determination of $\mathrm{k}$ values applying Equation (2).

$$
\mathrm{k}=\mathrm{m} \times 2.303
$$

Shelf life values (the time for $10 \%$ loss, $t_{90}$ ) and half-life (the time for $50 \%$ loss, $t_{1 / 2}$ ) were then calculated by means of Equations (3) and (4), respectively.

$$
\begin{aligned}
& t_{90}=0.105 / \mathrm{k} \\
& t_{1 / 2}=0.693 / \mathrm{k}
\end{aligned}
$$

\subsection{Antioxidant Activity (DPPH and FRAP)}

\subsubsection{DPPH (2,2-Diphenyl-1-picrylhydrazyl) Assay}

DPPH radical-scavenging assay is widely used to rapidly evaluate antioxidant capacity [40], and in particular it is ideal for phenolic compounds. This assay measures the hydrogen donation ability of an antioxidant to convert the stable DPPH free radical into 1,1-diphenyl-2-picrylhydrazyl, which is accompanied by a colorimetric reaction from deep-violet to light-yellow, which can be evaluated by measuring the percentage reduction of the absorbance of the solution at $517 \mathrm{~nm}$ after the radical reaction with the products to be tested. The percentage of radical scavenging capacity was calculated using Equation (5).

$$
\text { DPPH radical - scavenging capacity }(\%)=[1-(\mathrm{A} 1-\mathrm{A} 2) / \mathrm{A} 0] \times 100
$$


in which $\mathrm{A} 0$ is the absorbance of the control (without EA), A1 is the absorbance in the presence of the EA, and A2 is the absorbance without DPPH. To a methanol solution of DPPH (1.5 mL) 0.750 mL of EA (solution or NLCs in DMSO) at different concentrations were added. The absorbance at $517 \mathrm{~nm}$ was measured with a UV-Vis spectrophotometer (Jenway 7305 Spectrophotometer, VWR International Srl, Milan, Italy) according to a described procedure [41]. Results were expressed as $\mu$ mol Trolox equivalent/g of compounds.

\subsubsection{FRAP Assay}

The FRAP method is a quantitative assay for measuring the ferric ion reducing ability of plasma and is based on the reduction of ferric ions $\left(\mathrm{Fe}^{3+}\right)$ to ferrous ions $\left(\mathrm{Fe}^{2+}\right)$ under acidic conditions in the presence of 2,4,6-tripyridyl-s-triazine (TPTZ) [42]. In the presence of an antioxidant, the Fe ${ }^{3+}-\mathrm{TPTZ}^{3}$ complex is reduced to the ferrous form, corresponding to an intense blue coloration that is read to a fixed wavelength of the absorption maximum (593 nm). The antioxidant activity is given as $\mu \mathrm{mol}$ Trolox equivalent/g of compounds, as this standard was used to perform the calibration curves.

\subsection{In Vitro Diffusion Studies}

In vitro diffusion studies were performed using Franz-type diffusion cells supplied by Vetrotecnica (Padua, Italy) and associated to $0.45 \mu \mathrm{m}$ pore size nylon membranes (Merck Millipore, Milan, Italy). Before mounting onto a Franz cell (diameter being $1 \mathrm{~cm}$ ), nylon membranes were wetted in distilled water at room temperature for $30 \mathrm{~min}$. The exposed membrane area was $0.78 \mathrm{~cm}^{2}$. The receiving compartment contained $5 \mathrm{~mL}$ of a mixture of ethanol and phosphate buffer $60 \mathrm{mM}(30: 70, v / v)$ alternatively at $\mathrm{pH} 7.4$ or $\mathrm{pH}$ 5.5. The solution in the receiving compartment was stirred at $500 \mathrm{rpm}$ with a magnetic bar and maintained at $32 \pm 1{ }^{\circ} \mathrm{C}$ during the experiments $[31,43]$.

Then, $1 \mathrm{~mL}$ of each formulation was placed on the membrane in the donor compartment that was sealed to avoid evaporation. At predetermined time intervals comprised between 1 and $8 \mathrm{~h}$, $0.15 \mathrm{~mL}$ of receiving phase were withdrawn and EA content was evaluated by HPLC as above reported. Each removed sample volume was replaced with the same amount of fresh receiving phase. The EA concentrations were determined six times in independent experiments and the mean values \pm standard deviations were calculated. The mean values were then plotted as a function of time. The diffusion coefficients, computed from the linear portion of the accumulation curve, represent the experimentally observed fluxes (Jo). Normalized fluxes Jn were then calculated using Equation (6).

$$
\mathrm{Jn}=\mathrm{Jo} / \mathrm{C}
$$

where $\mathrm{C}$ is the EA concentration (in $\mathrm{mg} / \mathrm{mL}$ ) of the analyzed formulation.

\subsection{In Vitro MTT Test}

HaCaT cells were grown in Dulbecco's modified Eagle's medium (DMEM) high glucose, (Lonza, Milan, Italy), supplemented with 10\% FBS (fetal bovine serum), $100 \mathrm{U} / \mathrm{mL}$ penicillin, $100 \mu \mathrm{g} / \mathrm{mL}$ streptomycin, and $2 \mathrm{mM}$ L-glutamine. Cells were incubated at $37^{\circ} \mathrm{C}$ for $24 \mathrm{~h}$ in $95 \%$ air $/ 5 \% \mathrm{CO}_{2}$ until $80 \%$ confluence.

The different formulations, namely EA DMSO solution, both types of empty NLCs, NLC-EA1, and NLC-EA2, were dispersed in cell culture medium and diluted to reach EA concentrations ranging from 10 to $75 \mu \mathrm{M}$. Concerning empty-NLCs, they were added following the same dilution step used for NLC-EA in order to reach their same content in lipid nanoparticles within the wells.

Seeded cells were exposed to the selected formulations for $24 \mathrm{~h}$, afterwards the treatment was completely removed and $110 \mu \mathrm{L}$ of MTT $(0.5 \mathrm{mg} / \mathrm{mL})$ were added and incubated for $4 \mathrm{~h}$. To convert the MTT solution into a violet colored formazan, $100 \mu \mathrm{L}$ of DMSO were subsequently added and incubated for $15 \mathrm{~min}$. After shaking, the solution absorbance, proportional to the number of living 
cells, was measured using a spectrophotometer at $590 \mathrm{~nm}$ and, after subtracting background at $670 \mathrm{~nm}$, thus converted into percentage of viability.

\subsection{Data Analysis and Statistics}

Statistical analysis was performed by the analysis of variance (ANOVA). The level of significance was taken at $p$-values $<0.05$.

\section{Conclusions}

The low solubility of EA in aqueous solution involves very difficult administration. To ameliorate this, significant amounts of surfactants have to be used resulting in important toxicity in vivo. In this view the possibility to administrate EA using lipid nanoparticles could be very useful and interesting. It was demonstrated that the inclusion of EA within NLCs could improve the water solubility, allowing for a reduction of the dosage. Moreover, the maintenance of high antioxidant effect and low toxicity was evidenced for both types of NLC-EA, even if NLC-EA1 seems better than NLC-EA2.

It can be concluded that NLCs represent good strategies to encapsulate EA, although further studies aimed at deeply evaluating the absorption/diffusion of EA through the skin have to be carried out. For instance, in vitro studies should be carried out using natural epidermal stratum corneum and/or artificial membranes. In particular, in order to avoid the use of animals, a multi-layered membrane system consisting of a hydrophilic cellulose ester membrane sandwiched between two lipophilic Silastic ${ }^{\circledR}$ membranes should be used. In this way both the lipophilic-hydrophilic structure of human skin and the stratum corneum barrier properties can be satisfactorily reproduced, and in the meantime this system can be useful for simulating the dermal absorption of EA.

Furthermore, due to the awareness that simulations of human pharmacokinetic parameters and plasma concentration-time curves using in vitro extrapolation in vivo (IVIVE) and physiological based pharmacokinetics (PBPK) models are currently becoming very important and fundamental for the discovery and development of pharmacological processes, these models will be taken into consideration in the future and new experiments will be carried out in order to transpose some of the results obtained in this preliminary study.

Author Contributions: Conceptualization, R.C.; methodology, E.E. and R.C.; optimization and preparation of lipid nanoparticles, S.S.H. and M.S.; characterization of lipid nanoparticles, S.S.H., G.P., and M.S.; cryo-TEM experiments and discussion, M.D.; SAXS experiments and discussion, P.M.; DPPH and FRAP experiments and discussion, A.B.; original draft preparation, R.C.; writing-review and editing, R.C. and E.E. All authors have read and agreed to the published version of the manuscript.

Funding: This research was funded by University of Ferrara FIR2018 and FAR2018 and the APC was funded by MDPI.

Acknowledgments: The authors acknowledge Diamond Light Source for time on Beamline B21 under mail-in BAG session. Authors also acknowledge the kind support provided by Nikul Khunti for the work carried out at Diamond and provided by Francesca Falsone and Chiara Scanelli for technical issues. Unife FIR2018 and FAR2018 financially supported this study.

Conflicts of Interest: The authors declare no conflict of interest.

\section{References}

1. Malik, A.; Afaq, S.; Shahid, M.; Akhtar, K.; Assiri, A. Influence of ellagic acid on prostate cancer cell proliferation: A caspase-dependent pathway. Asian Pac. J. Trop. Med. 2011, 4, 550-555. [CrossRef]

2. Kaur, S.; Grover, I.S.; Kumar, S. Antimutagenic potential of ellagic acid isolated from Terminalia arjuna. Indian J. Exp. Biol. 1997, 35, 478-482.

3. Khanduja, K.L.; Gandhi, R.K.; Pathania, V.; Syal, N. Prevention of N Nitrosodiethylamine-Induced lung tumorigenesis by ellagic acid and quercetin in mice. Food Chem. Toxicol. 1999, 37, 313-318. [CrossRef]

4. Loarca-Piña, G.; Kuzmicky, P.A.; González de Mejía, E.; Kado, N.Y. Inhibitory effects of ellagic acid on the direct-acting mutagenicity of aflatoxin B1 in the Salmonella microsuspension assay. Mutat. Res. Fundam. Mol. Mech. Mutagenesis 1998, 398, 183-187. [CrossRef] 
5. Edderkaoui, M.; Odinokova, I.; Ono, I.; Gukovsky, I.; Go, V.L.W.; Pandol, S.J.; Gukovskaya, A.S. Ellagic acid induces apoptosis through inhibition of nuclear factor $\mathrm{kB}$ in pancreatic cancer cells. World J. Gastoenterol. 2008, 14, 3672-3680. [CrossRef]

6. Losso, J.N.; Bansode, R.R.; Trappey, A.; Bawadi, H.A. Truax R: In vitro antiproliferative activities of ellagic acid. J. Nutr. Biochem. 2014, 15, 672-678. [CrossRef]

7. Hagiwara, Y.; Kasukabe, T.; Kaneko, Y.; Niitsu, N.; Okabe-Kado, J. Ellagic acid, a natural polyphenolic compound induces apoptosis and potentiates retinoic acid-induced differentiation of human leukemia HL-60 cells. Int. J. Hematol. 2010, 92, 136-143. [CrossRef]

8. Fjaeraa, C.; Nanberg, E. Effect of ellagic acid on proliferation, cell adhesion and apoptosis in SH-SY5Y human neuroblastoma cells. Biomed. Pharmacother. 2009, 63, 254-261. [CrossRef]

9. Gonzalez-Sarrias, A.; Espin, J.-C.; Tomas-Barberan, F.A. Garcia-Conesa M-T Gene expression, cell cycle arrest and MAPK signaling regulation in Caco-2 cells exposed to ellagic acid and its metabolites, urolithins. Mol. Nutr. Food Res. 2009, 53, 686-698. [CrossRef]

10. Han, D.H.; Lee, M.J.; Kim, J.H. Antioxidant and apoptosis-inducing activities of ellagic acid. Anticancer Res. 2006, 26, 3601-3606.

11. Seeram, N.P.; Adams, L.S.; Henning, S.M.; Niu, Y.; Zhang, Y.; Nair, M.G.; Heber, D. In vitro antiproliferative, apoptotic and antioxidant activities of punicalagin, ellagic acid and a total pomegranate tannin extract are enhanced in combination with other polyphenols as found in pomegranate juice. J. Nutr. Biochem. 2005, 16, 360-367. [CrossRef]

12. Rosillo, M.A.; Sanchez-Hidalgo, M.; Cárdeno, A.; Alarcón De La Lastra, C. Protective effect of ellagic acid, a natural polyphenolic compound, in a murine model of Crohn's disease. Biochem. Pharmacol. 2011, 82, 737-745. [CrossRef]

13. Landete, J.M. Ellagitannins, ellagic acid and their derived metabolites: A review about source, metabolism, functions and health. Food Res. Int. 2011, 44, 1150-1160. [CrossRef]

14. Larrosa, M.; Garcia-Conesa, M.T.; Espin, J.C.; Tomas-Barberan, F.A. Ellagitannins, ellagic acid and vascular health. Mol. Asp. Med. 2010, 31, 513-539. [CrossRef]

15. Bala, I.; Bhardwaj, V.; Hariharan, S.; Kumar, M.N.V. Analytical methods for assay of ellagic acid and its solubility studies. J. Pharmaceut. Biomed. 2006, 40, 206-209. [CrossRef]

16. Chavda, H.V.; Patel, C.N.; Anand, I.S. Biopharmaceutics classification system. Syst. Rev. Pharm. 2010, 1, 62-68. [CrossRef]

17. Seeram, N.P.; Lee, R.; Heber, D. Bioavailability of ellagic acid in human plasma after consumption of ellagitannins from pomegranate (Punica granatum L.) juice. Clin. Chim. Acta 2004, 348, 63-68. [CrossRef]

18. Lim, S.J.; Lee, M.K.; Kim, C.K. Altered chemical and biological activities of all-trans retinoic acid incorporated in solid lipid nanoparticle powders. J. Control. Rel. 2004, 100, 53-61. [CrossRef]

19. Arias, J.L.; Clares, B.; Morales, M.E.; Gallardo, V.; Ruiz, M.A. Lipid-based drug delivery systems for cancer treatment. Curr. Drug Targets 2011, 8, 1151-1165. [CrossRef]

20. Ekambaram, P.; Abdul Hasan Sathali, A.; Priyanka, K. Solid lipid nanoparticles: A review. Sci. Rev. Chem. Commun. 2012, 2, 80-102.

21. Doktorovova, S.; Souto, E.B.; Silva, A.M. Nanotoxicology applied to solid lipid nanoparticles and nanostructured lipid carriers - A systematic review of in vitro data. Eur. J. Pharm. Biopharm. 2014, 87, 1-18. [CrossRef] [PubMed]

22. Hernqvist, L. Crystal structures of fats and fatty acids. In Crystallization and Polymorphism of Fats and Fatty Acids; Garti, N., Sato, K., Eds.; Marcel Dekker: New York, NY, USA, 1988; pp. 97-138.

23. Esposito, E.; Sguizzato, M.; Drechsler, M.; Mariani, P.; Carducci, F.; Nastruzzi, C.; Valacchi, G.; Cortesi, R. Lipid nanostructures for antioxidant delivery: A comparative preformulation study. Beilstein J. Nanotechnol. 2019, 10, 1789-1801. [CrossRef] [PubMed]

24. Esposito, E.; Sguizzato, M.; Drechsler, M.; Mariani, P.; Carducci, F.; Nastruzzi, C.; Cortesi, R. Progesterone lipid nanoparticles: Scaling up and in vivo human study. Eur. J. Pharm. Biopharm. 2017, 119, 437-446. [CrossRef] [PubMed]

25. Sguizzato, M.; Cortesi, R.; Gallerani, E.; Drechsler, M.; Marvelli, L.; Mariani, P.; Carducci, F.; Gavioli, R.; Esposito, E.; Bergamini, P. Solid lipid nanoparticles for the delivery of 1,3,5-triaza-7-phosphaadamantane (PTA) platinum (II) carboxylates. Mater. Sci. Eng. C Mater. Biol. Appl. 2017, 74, 357-364. [CrossRef] 
26. Badhani, B.; Sharmaa, N.; Kakkar, R. Gallic acid: A versatile antioxidant with promising therapeutic and industrial applications. R. Soc. Chem. Adv. 2015, 5, 27540-27557. [CrossRef]

27. Lo, C.; Lai, T.Y.; Yang, J.H.; Yang, J.S.; Ma, Y.S.; Weng, S.W.; Chen, Y.Y.; Lin, J.G.; Chung, J.G. Gallic acid induces apoptosis in A375.S2 human melanoma cells through caspase-dependent and -independent pathways. Int. J. Oncol. 2010, 37, 377-385.

28. Yang, D.J.; Moh, S.H.; Son, D.H.; You, S.; Kinyua, A.W.; Ko, C.M.; Song, M.; Yeo, J.; Choi, Y.H.; Kim, K.W. Gallic acid promotes wound healing in normal and hyperglucidic conditions. Molecules 2016, $21,899$. [CrossRef]

29. Soares, J.S.; Dins, T.C.P.; Cunha, A.P.; Almerida, L.M. Antioxidant activity of some extracts of Thymus zygis. Free Radic. Res. 1997, 26, 469-472. [CrossRef]

30. Cao, G.; Alessio, H.M.; Cutler, R.G. Oxygen-radical absorbance capacity assay for antioxidants. Free Radic. Biol. Med. 1993, 14, 303-311. [CrossRef]

31. Siewert, M.; Dressman, J.; Brown, C.K.; Shah, V.P. FIP/AAPS guidelines to dissolution/in vitro release testing of novel/special dosage forms. AAPS PharmSciTech 2003, 4, 7. [CrossRef]

32. Santos, I.S.; Ponte, B.M.; Boonme, P.; Silva, A.M.; Souto, E.B. Nanoencapsulation of polyphenols for protective effect against colon-rectal cancer. Biotechnol. Adv. 2013, 31, 514-523.

33. Lambers, H.; Piessens, S.; Bloem, A.; Pronk, H.; Finkel, P. Natural skin surface pH is on average below 5, which is beneficial for its resident flora. Int. J. Cosmet. Sci. 2006, 28, 359-370. [CrossRef]

34. Salamanca, C.H.; Barrera-Ocampo, A.; Lasso, J.C.; Camacho, N.; Yarce, C.J. Franz diffusion cell approach for pre-formulation characterisation of ketoprofen semi-solid dosage forms. Pharmaceutics 2018, 10, 148. [CrossRef]

35. Spagnol, C.M.; Zaera, A.M.; Borges Isaac, V.L.; Corrêa, M.A.; Nunes Salgado, H.R. Release and permeation profiles of spray-dried chitosan microparticles containing caffeic acid. Saudi Pharm. J. 2018, 26, 410-415. [CrossRef]

36. Pranitha, A.; Lakshmi, P.K. Effect of $\mathrm{pH}$ on weakly acidic and basic model drugs and determination of their ex vivo transdermal permeation routes. Braz. J. Pharm. Sci. 2018, 54, e00070.

37. Svilenov, H.; Tzachev, C. Solid Lipid Nanoparticles-A Promising Drug Delivery System; One Central Press: Manchester, UK, 2014; pp. 187-237.

38. Pecora, R. Dynamic Light Scattering measurement of nanometer particles in liquids. J. Nanoparticle Res. 2000, 2, 123-131. [CrossRef]

39. Pugh, W.J. Kinetics of product stability. In The Design and Manufacture of the Medicines, 3rd ed.; Churchill Livingstone Elsevier: London, UK, 2007; pp. 99-107.

40. Blois, M.S. Antioxidant determinations by the use of a stable free radical. Nature 1958, 181, 1199-1200. [CrossRef]

41. Wang, M.; Li, J.; Rangarajan, M.; Shao, Y.; La Voie, E.J.; Huang, T.-C.; Ho, C.T. Antioxidative phenolic compound from sage (salvia officinalis). J. Agric. Food Chem. 1998, 46, 4868-4873. [CrossRef]

42. Benzie, I.F.F.; Strain, J.J. The ferric reducing ability of plasma (FRAP) as a measure of "antioxidant power": The FRAP assay. Anal. Biochem. 1996, 239, 70-76. [CrossRef]

43. Andrade, L.M.; de Fátima Reis, C.; Maione-Silva, L.; Anjos, J.L.; Alonso, A.; Serpa, R.C.; Marreto, R.N.; Lima, E.M.; Taveira, S.F. Impact of lipid dynamic behavior on physical stability, in vitro release and skin permeation of genistein-loaded lipid nanoparticles. Eur. J. Pharm. Biopharm. 2014, 88, 40-47. [CrossRef]

Sample Availability: Samples are available from the authors. 